\title{
Les nouveaux espaces publics chez les Yucuna d'Amazonie colombienne
}

The new public spaces of the Yucuna Indians (Colombian Amazon)

Los nuevos espacios públicos de los yucunas de la Amazonia colombiana

Laurent Fontaine

\section{OpenEdition}

Journals

Édition électronique

URL : https://journals.openedition.org/jsa/12612

DOI : 10.4000/jsa. 12612

ISSN : 1957-7842

\section{Éditeur}

Société des américanistes

Édition imprimée

Date de publication : 15 septembre 2013

Pagination : $77-104$

ISSN : 0037-9174

Référence électronique

Laurent Fontaine, "Les nouveaux espaces publics chez les Yucuna d'Amazonie colombienne ", Journal de la Société des américanistes [En ligne], 99-1 | 2013, mis en ligne le 01 mars 2016, consulté le 02 septembre 2022. URL : http://journals.openedition.org/jsa/12612 ; DOI : https://doi.org/10.4000/ jsa.12612 


\title{
LES NOUVEAUX ESPACES PUBLICS CHEZ LES YUCUNA D'AMAZONIE COLOMBIENNE
}

\author{
Laurent FONTAINE *
}

Depuis le début $\mathrm{du} \mathrm{xx}^{\mathrm{e}}$ siècle, les contacts réguliers avec certains acteurs extérieurs nouveaux ont progressivement amené les Yucuna à faire l'expérience de nouveaux espaces publics, en transformant définitivement leur organisation sociale. Cet article passe en revue l'histoire de ces espaces. L'analyse des lieux de rassemblement traditionnels montre qu'ils se distinguent des espaces publics modernes. L'entrée en scène des exploiteurs de caoutchouc, des représentants de l'État et des missionnaires a conduit les communautés yucuna à s'ouvrir à la société englobante. Les années 1970 ont été marquées par l'apparition des assemblées indigènes, qui constituent sans doute le cadre d'interaction le plus représentatif des espaces publics modernes intégrés au sein des communautés. [Mots-clés : espace public, contexte, Yucuna, Amazonie, Colombie.]

The new public spaces of the Yucuna Indians (Colombian Amazon). Since the beginning of the twentieth century, the regular contacts of the Yucuna Indians with some new external actors have gradually led them to experience new public spaces, permanently transforming their social organization. This article reviews the history of these spaces. The analysis of traditional gathering areas shows that they are distinguishable from modern public spaces. It is only with the entrance of the rubber exploiters, state officials and missionaries, that the Yucuna communities were forced to open up to the surrounding society. The 1970's were then marked by the emergence of the indigenous assemblies that are probably the areas of interaction which are the most representative of modern public spaces integrated in communities. [Key words : public space, context, Yucuna, Amazon, Colombia.]

Los nuevos espacios públicos de los yucunas de la Amazonia colombiana. Desde el comienzo del siglo $\mathrm{xx}$, los contactos regulares de los indígenas yucunas con unos nuevos actores exteriores los han conducido progresivamente a experimentar nuevos espacios públicos, transformando definitivamente su organización social. Este artículo examina la historia de estos espacios. El análisis de las áreas de agrupación tradicional muestra que se distinguen de los espacios públicos modernos. Es únicamente cuando llegaron los caucheros, corregidores y misioneros, que las comunidades yucunas fueron

* Membre du Laboratoire des langues et civilisations à tradition orale (Lacito, UMR 7107 du CNRS), 7 rue Guy Môquet (bâtiment D), F-94801 Villejuif Cedex [fontaine@vjf.cnrs.fr].

Journal de la Société des Américanistes, 2013, 99-1, pp. 77-104. C Société des Américanistes. 
obligadas a abrirse hacia la sociedad englobante. Después, los años 1970 fueron marcados por la emergencia de asambleas indígenas, las cuales constituyen probablemente el marco de interacción más representativo de los espacios públicos modernos integrados dentro de las comunidades. [Palabras claves : espacio público, contexto, yucunas, Amazonia, Colombia.]

Dans cet article, nous considèrerons la notion d' " espace public » au sens d'Habermas (1978), en tant que lieu de « publicité », c'est-à-dire de propagation des informations, des idées et des débats. Rappelons que, selon cet auteur, les espaces publics furent historiquement institués et promus par la société bourgeoise dès le XVIII ${ }^{\mathrm{e}}$ siècle.

Si l'on veut appliquer cette notion d'« espace public» à une société traditionnelle comme celle des Yucuna, il faut d'abord tenter de répondre à deux questions anthropologiques ${ }^{1}$. La première est de savoir si la société considérée n'aurait pas élaboré « quelque chose » - une conception, une pratique, un mode d'organisation - qui ressemble à un « espace public ». Si une telle "chose» existait, cela permettrait d'examiner en détail un certain nombre de caractéristiques et de fonctionnements communs à cet objet d'étude dans différentes sociétés et de construire une base comparative. La seconde question porte sur les influences que notre société peut avoir sur la société yucuna à travers cet objet d'étude. De quelles manières celui-ci peut-il la transformer? En considérant ces deux questions, l'ethnologue des sociétés traditionnelles contemporaines (appartenant pleinement à notre temps) éprouve souvent des difficultés à distinguer, au sein de son objet, ce qui est originel à la culture étudiée et ce qui a été introduit par la société dominante (voire par les ethnologues eux-mêmes).

Durant la seconde moitié $\mathrm{du} \mathrm{xx}^{\mathrm{e}}$ siècle, les Yucuna ont commencé à admettre de nouvelles formes d'espace public, introduites par les Blancs (notamment par les missionnaires, les linguistes et les ethnologues) ${ }^{2}$. Aujourd'hui, ils savent tout à fait les reproduire au sein de leur organisation sociale, que ce soit de manière interne ou vis-à-vis de l'extérieur.

Il existe d'autres types de situations communicatives que l'on peut qualifier de traditionnelles et qui se caractérisent, contrairement aux espaces publics récents, par un accès très limité tant au niveau de l'émission que de la réception des énoncés. Dans ces différents cadres d'énonciation, le champ de diffusion des informations est restreint, les paroles sont le plus souvent privées ou secrètes ${ }^{3}$ (réservées à certaines personnes). Certaines situations précises (comme les rassemblements de guerre ou les cérémonies dansantes) constituent des cas intéressants susceptibles de faire exception, c'est pourquoi je les examinerai du point de vue de leurs différentes conditions d'énonciation pour mieux étudier dans quelle mesure elles se distinguent de celles des espaces publics modernes. Une fois que nous aurons précisé ces distinctions, nous serons mieux disposés 
pour retracer historiquement la transformation progressive des espaces publics et pour analyser leur participation au changement de l'organisation sociale.

\section{LES ESPACES DE RASSEMBLEMENT TRADITIONNELS}

Si l'on veut se faire une idée des formes d'espace public existant chez les Yucuna avant que ceux-ci n'apprennent à reproduire celles des Blancs, il convient d'examiner en quoi et selon quels critères certains lieux traditionnels peuvent être apparentés, à divers degrés, à des espaces publics modernes, notamment dans certains types de contexte.

Chez les Yucuna, la maloca (grande maison plurifamiliale et festive) est l'espace qui partage le plus de points communs avec les espaces publics de nos sociétés industrialisées. Elle est un espace social ${ }^{4}$ d'usage collectif, pour ses nombreux résidents, les familles voisines, les visiteurs ou les invités. Elle est aussi un espace de rassemblement régulier, particulièrement en cas de conflit ou de cérémonie, comme nous allons le voir. Elle est enfin un lieu permettant de parler et d'être entendu au sein d'une foule ${ }^{5}$ et, donc, de diffuser des informations à beaucoup d'auditeurs. Mais, à la différence des espaces publics modernes (au sens d'Habermas), on ne peut pas y débattre, avancer des idées nouvelles ou laisser circuler et manipuler les informations qui y sont produites au-delà de certains groupes particuliers et restreints. En outre, l'autorité traditionnelle ne s'appuie jamais sur le consensus d'une majorité au sein d'une population donnée (en cela, elle ne peut être légitime au-delà de son clan relativement restreint). L'autorité traditionnelle - peu soucieuse de l'approbation au-delà du clan - s'appuie, au contraire, sur des paroles prononcées en petits comités et, même, essentiellement, sur des paroles secrètes (notamment celles de l'initiation rituelle et du chamanisme). Étant donné ces caractéristiques propres aux Yucuna, comme à beaucoup d'autres sociétés traditionnelles, de telles sociétés pourraient être qualifiées de « sociétés sans publicité » ou de « sociétés du secret " ${ }^{6}$, par opposition à nos sociétés modernes que l'on appellerait « sociétés de la publicité ».

Pour aborder plus précisément des situations de communication traditionnelles qui peuvent avoir des traits communs avec les espaces publics modernes, j'examinerai celles qui restreignent le moins le nombre de leurs auditeurs potentiels. En effet, contrairement aux situations de communication publique moderne, toute situation de communication traditionnelle yucuna se doit de connaître ses auditeurs et de choisir, parmi eux, les destinataires aptes à recevoir l'information. Aucune parole, aucun message ne peut donc être diffusé ouvertement. La qualification du candidat à la réception de l'information dépend toujours de toute une série de conditions contextuelles dans lesquelles entrent en jeu, le plus souvent, ses caractéristiques propres (sexe, âge, statut), ses relations avec le locuteur (parenté, clan), son respect des règles sociales (division des tâches, 
rituels) ou, encore, le type de scénario engagé avec le locuteur (complémentarité des rôles au sein d'une activité commune). Autant dire que, traditionnellement, la parole yucuna est toujours obligatoirement dirigée à l'attention d'un certain groupe ou d'une certaine personne, clairement identifiée. Son auditoire n'est jamais anonyme.

Pour les Yucuna, les situations communicatives visant un auditoire relativement large, même s'il s'agit toujours d'un groupe déterminé et connu, sont des occasions de « se regrouper » (jawakákajo) ${ }^{7}$. Il s'agit principalement des rassemblements liés aux conflits et des cérémonies.

\section{Les rassemblements liés aux conflits}

À en croire les différentes sources historiques, il semblerait que les rassemblements liés aux conflits étaient autrefois relativement courants en raison du nombre de guerres intertribales qui avaient lieu dans la région et qui ont été repérées depuis le XVII ${ }^{\mathrm{e}}$ siècle (Pineda Camacho 1985). De tels conflits se seraient même grandement intensifiés en raison du trafic d'esclaves, comme Crevaux, un voyageur français de la seconde moitié du XIX ${ }^{\mathrm{e}}$ siècle, le raconte (Llanos Vargas et Pineda Camacho 1982), et auraient eu un "grand impact démographique » en entraînant une chute importante de la population du Bas Caqueta, autrefois très peuplé (Van der Hammen 1992, pp. 27-28 ; Karadimas 1997, pp. 39-40).

Les ralliements de guerre sont largement représentés dans les mythes et autres récits ${ }^{8}$. Dans ceux que j'ai pu transcrire et traduire ${ }^{9}$, le verbe jawakákajo (« se regrouper ») renvoie le plus souvent à des ralliements stratégiques liés à des contextes de guerre. Examinons quelques-unes des scènes représentées.

a) Dans le mythe du grillon contre la chauve-souris, cette dernière part en guerre contre le grillon pour se venger d'une offense dite à sa femme à son sujet. Mais, après l'échec cuisant d'une première bataille, la chauve-souris « rassembla un grand nombre de ses frères (kajrú rawakáchiyaka rika'jná) dans sa maloca » pour préparer sa revanche.

b) Dans le mythe du jaguar Jiyánama, le père de celui-ci est d'abord tué par son oncle paternel, ce qui incite sa mère à se réfugier avec Jiyánama, encore enfant, chez l'Ancêtre des vers de terre. Mais, plus tard, le jeune Jiyánama s'aperçoit que les poissons de son père adoptif sont tirés d'une écœurante décomposition de ce dernier dans la rivière. Alors il décide de fuir avec sa mère. Et l'Ancêtre lombric "rassembla ses clans" (rawakáchiya rika'ána) pour partir à leur poursuite.

c) Dans le mythe de Kuwáiri, celui-ci s'aperçoit que sa sœur s'est fait engrosser par le père des serpents. Lors d'une cueillette en forêt, il la tue en faisant tomber sur elle un énorme régime de milpeso (Jessenia $s p$.), ce qui fait éclater son ventre en laissant fuir une multitude de petits serpents qu'il ne parvient pas à exterminer. Plus tard, les serpents "se rassemblèrent entre eux" (nawakáchiyo pekowaka) et décidèrent de venger leur mère. Ils préparèrent alors une énorme marmite de curare pour tuer Kuwáiri. 
Si l'on admet que la plupart des événements ordinaires décrits dans les mythes s'inspirent de pratiques sociales réelles (Propp 1983), une bonne part des regroupements traditionnels semblent avoir été des « rassemblements militaires » permettant à un chef de guerre d'organiser ses attaques à partir de sa maloca, notamment dans des situations de revanche guerrière (a), de poursuite (b) ou de vendetta (c). Pour chacun de ces rassemblements, les chefs envoyaient des émissaires chez leurs frères et alliés à qui ils demandaient de transmettre formellement des paroles cérémonielles qui avaient pour fonction d'annoncer les raisons de leurs griefs et de les solliciter au plus vite. Puis, une fois qu'ils étaient arrivés dans la maloca du chef, les hommes attendaient généralement le soir ${ }^{10}$ pour discuter en conciliabule, particulièrement pour évaluer entre eux la gravité des événements, prendre des décisions, élaborer des stratégies ou, encore, faire de la divination de guerre ${ }^{11}$. On comprendra que de tels espaces de rassemblement ne sont jamais vraiment ouverts (même s'ils appellent à la communication à l'intérieur d'un groupe), car les discussions et décisions prises en temps de guerre doivent absolument rester secrètes au sein du groupe pour ne pas alerter l'ennemi.

Parmi les situations de conflit suscitant des regroupements et des déclarations au sein d'un groupe, il peut y avoir également la préparation d'une fuite, ce qui est le cas dans certaines histoires de guerre, lorsqu'un repli stratégique est nécessaire. L'histoire de Ka'marí présente ce type de situation.

Pour préciser davantage les caractéristiques propres aux rassemblements dans des situations de conflits, je mentionnerai le cas particulier du mythe de Kanumá, le premier homme (Herrera 1975a, 1975b ; Schackt 1994, pp. 29-36 ; Fontaine 2010b). Au début, Kanumá est au service (domestique) des Namatu, les premières femmes, douées de pouvoirs surnaturels. Mais, un jour, il leur vole les flûtes sacrées du Yurupari ${ }^{12}$ qu'elles lui avaient toujours interdit de voir. Le soir, elles se rassemblent (nawakicho) dans la maloca pour parler entre elles et considèrent qu'elles ne peuvent plus vivre avec lui. Elles fuient en abandonnant Kanumá. S'ensuit alors une course poursuite du haut du fleuve Miriti jusqu'à l'embouchure de l'Amazone.

Cet épisode du mythe qui traite des différences sociales entre hommes et femmes est intéressant, particulièrement parce que les rapports de genre sont inversés : les femmes se rassemblent entre elles pour parler et prendre des décisions collectives. Ce qu'elles ne peuvent précisément pas faire car, dans la société traditionnelle, toute parole exprimée devant l'ensemble du groupe est obligatoirement masculine. À aucun moment dans un rassemblement, elles ne peuvent participer aux prises de décisions collectives pour régler des conflits, ce qui les empêche de traiter de leurs conditions spécifiques ou de leurs rapports aux hommes, contrairement à ces derniers, notamment lors des rites de Yurupari où les femmes sont tenues à l'écart. L'espace de rassemblement traditionnel est, donc, avant tout, un rapport social dans lequel les droits et les devoirs des uns et des autres sont strictement réglementés. 
Un dernier motif de rassemblement lié au conflit est décrit dans l'histoire de Ka'marí, l'ancêtre éponyme des Matapi ${ }^{13}$. Après maintes batailles entre le clan de Ka'marí et celui de son frère Junimari, ce dernier est obligé de regrouper ses compagnons pour fuir et demander refuge auprès de plusieurs tribus habitant à proximité du fleuve Apaporis. L'un des chefs locaux organise plusieurs rassemblements avec tous les groupes voisins pour prendre une décision à propos de cette demande d'asile. Après un accueil hostile et menaçant, ces groupes finiront par les accepter et, même, par les aider concrètement en leur prêtant une partie de leurs terres cultivées et en participant à leurs travaux d'installation (maloca, jardins).

\section{Les rassemblements cérémoniels}

Les cérémonies sont, comme les conflits, des moments de rassemblement pour les gens. Elles sont principalement de deux types : les enterrements et les fêtes. De tels rassemblements s'apparentent aux espaces publics modernes dans la mesure où ils tendent à rapprocher certaines personnes ou certaines connaissances et prennent soin d'écarter tout intrus indésirable. De plus, certains messages peuvent être diffusés " publiquement », c'est-à-dire en dehors des rassemblements eux-mêmes, notamment pour déclarer, officialiser ou sacraliser le moment de la cérémonie. Pour les cérémonies funéraires, cela est d'autant plus vrai qu'il est assez difficile pour un ethnologue d'y participer, s'il n'est pas lui-même un ami intime du défunt ou de sa famille. Selon les informations que j'ai pu recueillir, il semble que les normes soient assez strictes et éprouvantes, puisqu'il s'agirait de «pleurer» (iyákaje) avec les proches du défunt pendant trois journées successives. Les vieillards, quant à eux, chanteraient sur un pas de danses sacrées en portant des costumes spéciaux ne pouvant pas être utilisés à d'autres moments. En outre, il est formellement interdit de rire ou d'émettre le moindre ricanement pendant toute la durée du rituel, pour ne pas déclencher la colère et la malédiction de Ñámatu, la femme mythique qui enseigna les danses de deuil aux humains ${ }^{14}$.

Signalons néanmoins qu'une cérémonie funéraire yucuna n'est pas seulement une situation dans laquelle tout ce qui est pratiqué est uniquement destiné aux participants autorisés à se réunir autour du défunt, elle est aussi l'occasion d'exécuter un acte entièrement «public » : l'annonce du décès au son des tambours manguaré ${ }^{15}$. En effet, selon le type de rythme qui est martelé, chacun peut en principe reconnaître, quelle que soit son identité, la situation funéraire et, peut-être, même le statut du défunt ${ }^{16}$. Contrairement à la plupart des autres informations, l'annonce d'un décès n'a généralement rien de secret, surtout si elle a été manifestée (ou rendue publique) au son des manguaré. Chacun est libre de diffuser la nouvelle, à condition de parler du mort avec respect. Dès lors, tous les êtres, humains ou non humains, sont conviés à pleurer en même temps que les endeuillés. Selon des anciens, plusieurs chants de deuil auraient été transmis aux humains par certaines espèces animales (notamment les poissons). 
De ce fait, tous les animaux seraient aptes à les entendre, ce qui les inciterait à faire silence et à pleurer également.

Pour les cérémonies festives, les rassemblements sont, là encore, réservés aux seuls groupes des invités. Un maître de maloca organisant une fête se doit de faire savoir qui il souhaite réunir en transmettant ses paroles cérémonielles d'invitation (yukupéra'kaje) à chaque chef local par l'intermédiaire de ses émissaires. Dans le mythe de Kawáirimi (Jacopin 1981), lorsque celui-ci rencontre un groupe de toucans et de loriots faisant route vers la fête des animaux, il ne peut tenter de s'y rendre avec eux sans leur demander leur avis. Ce à quoi ils répondent : « Je ne sais pas, compagnon. Je ne sais pas si c'est possible, si c'est bien que tu viennes avec nous... Ce sont tes ennemis, tu ne peux pas nous accompagner » (ibid., p. 96). Plus tard, l'intrusion du héros ne manquera pas de susciter la colère du singe capucin et son désir de le tuer (ibid., p. 97).

De même, dans le mythe du jaguar Jiyánama, lorsque le jeune héros rencontre les hiboux qui vont danser pour son oncle jaguar, ceux-ci s'apprêtent à demander la fille de l'oncle jaguar pour le fils de leur chef. Jiyánama propose alors aux hiboux de les accompagner, mais ils refusent d'abord catégoriquement, en lui répondant qu'il pourrait séduire la fille ou, encore, se faire tuer par son oncle jaguar (qui est déjà le meurtrier du père de Jiyánama). Les hiboux finiront par accepter mais, évidemment, le regretteront...

Outre ces restrictions d'accès aux participants, toute cérémonie dansante restreint également les possibilités d'énonciation, d'écoute ou de compréhension. D'un côté, certains genres de paroles ne peuvent être énoncés que par certains locuteurs. De l'autre, ils ne peuvent être entendus que par certains destinataires. Au sein d'un groupe, et pour chaque genre de parole, il est donc souvent pertinent de distinguer l'auditoire proprement dit, autorisé à écouter ou obligé d'entendre, du reste du groupe qui n'y est pas autorisé, ni obligé. Ainsi, pour un auditoire donné, il est parfois utile de préciser ce que j'appelle le «non-auditoire », c'est-à-dire l'ensemble des individus présents dans une situation donnée auxquels un message n'est pas adressé (soit l'ensemble complémentaire de l'auditoire).

Pour chaque genre de parole énoncé devant un grand nombre de personnes lors d'une cérémonie, on peut distinguer plusieurs ensembles de sujets qui sont plus ou moins restreints et qui se différencient par leur type de participation supposée dans la communication. Ainsi, des plaisanteries à allusions sexuelles sont fréquemment dites à voix haute par des hommes devant la foule, presque toujours à l'attention d'autres hommes. Les grossièretés sont, en quelque sorte, ritualisées ou prévues et participent aux plaisirs de la fête. Mais il est très rare qu'elles soient dites à des femmes car cela est offensant pour elles, surtout devant de nombreuses personnes. Ni elles - ni d'ailleurs les enfants - ne sont censées en rire si elles veulent préserver une certaine dignité ou respectabilité (leur sérieux étant souvent associé au courage et au travail). 
Notons bien que, pour une anthropologie qui souhaite définir les conditions de sa propre pragmatique (Rumsey 2009 ; Severi 2009, p. 14), cette distinction entre auditoire et non-auditoire est cruciale. En effet, c'est souvent le fait que des paroles puissent diviser parmi les personnes présentes, entre celles qui sont obligées ou non de les écouter et celles qui sont autorisées ou non à les entendre, qui rend possible la superposition de plusieurs cadres d'interaction au sein d'un même espace de perception. Cet effet particulier des actes de parole (Austin 1970) qui divisent les cadres d'interaction en excluant certains interlocuteurs à l'intérieur d'un même contexte est évidemment l'une des raisons pour lesquelles les principes de coopération de Grice (1979) ${ }^{17}$ et de pertinence de Sperber et Wilson (1989) ${ }^{18}$ ne peuvent pas être utilisés dans bon nombre de situations d'interlocution étudiées par les ethnologues (notamment dans les rituels) pour faire des inférences. Pour envisager les inférences qui peuvent être réalisées par les auditeurs potentiels, il est donc nécessaire de dégager, au préalable, les règles propres à chaque type de contexte (Fontaine 2001).

À l'opposé des paroles peu valorisées ou valorisantes qui viennent d'être mentionnées, d'autres formes d'énonciation à large auditoire constituent l'essentiel de ce qui doit absolument être dit lors d'une fête. Il s'agit des dialogues cérémoniels et des incantations. Si les dialogues cérémoniels sont très importants, c'est certainement parce qu'ils explicitent les nombreuses conventions propres à la fête, notamment celles pour lesquelles elle a été engagée et en vertu desquelles chacun est en droit d'attendre un ensemble de devoirs implicitement admis par les autres participants du seul fait de leur présence et de leur coopération au rituel. Seuls les initiés assumant certaines charges lors des cérémonies (maître de maloca, chanteurs, chamanes ou soigneurs) sont autorisés à connaître ${ }^{19}$ et à débiter ces paroles dans un langage très formel et particulier à leurs langues spécifiques, si bien que les non-initiés, les femmes et les enfants ne peuvent généralement les comprendre. Les dialogues cérémoniels se font toujours par paires de partenaires. Ils sont dits à deux au moment des salutations d'ouverture, de milieu et de clôture de la fête, principalement entre le maître de maloca et chacun des invités assumant une charge cérémonielle importante. Ils sont prononcés en deux rangs face à face (celui du clan des hôtes et celui des invités) lors des offrandes de nourriture et de coca. Chaque rang débite ses paroles, en même temps, d'un côté, puis de l'autre, plusieurs fois d'affilée (Fontaine 2001, p. 537).

Chaque initié peut très bien formuler ses paroles dans une langue et se voir répondre dans une autre langue, sans que ni l'un, ni l'autre des deux locuteurs n'aient besoin de comprendre la langue de l'autre. L'essentiel n'est pas que ces paroles soient intelligibles pour celui d'en face, mais qu'elles soient dites correctement ; de cette façon elles pourront être implicitement reconnues, non seulement par les autres initiés qui les entendent, mais surtout par les ancêtres qui en furent à l'origine ou qui les transmirent. Car, du point de vue des anciens, c'est essentiellement pour eux qu'elles sont rappelées. Paradoxalement donc, peu 
importe que les paroles cérémonielles soient peu comprises ou de portée limitée (elles sont criées en même temps, dans des langues différentes, au milieu du brouhaha festif) ${ }^{20}$, l'important c'est qu'elles puissent - en principe - être écoutées par l'ensemble des ancêtres pour juger de leur valeur.

Ce qui précède est encore plus vrai dans le cas des incantations des soigneurs (Fontaine 2011), puisqu'elles ne sont jamais énoncées à voix haute devant la foule, contrairement aux autres paroles que je viens de présenter, mais seulement murmurées $^{21}$. Ces paroles sont alors exclusivement destinées aux divinités, esprits et ancêtres, afin d'obtenir leur accord et leur bienveillance pour la cérémonie. Comme pour toute formulation de parole rituelle supposée être écoutée au-delà du monde visible, le soigneur se doit de détenir de la coca en quantité suffisante pour en offrir symboliquement à toutes les entités surnaturelles invitées à la fête. De même se devra-t-il de leur dédier tout autre " compagnon » (wáke'weji), qu'il s'agisse des aliments spirituels indispensables au rituel (coca à mâcher, tabac, alcool) ou de toute autre nourriture (galette de manioc, viande, poisson). De plus, pour que les danses puissent être réalisées, il en demandera la permission à leurs maîtres légitimes (par exemple, Je'chú « le Ciel »).

De même que pour les autres paroles rituelles (dialogues cérémoniels, chants, mythes), les incantations chamaniques sont supposées soumettre leurs locuteurs à des responsabilités très lourdes, car toute faute est susceptible d'entraîner la colère des entités (ancêtres, esprits) qui en sont les garants et donc de déclencher leurs malédictions (Fontaine 2010a). Ainsi, des erreurs, oublis (de certains noms d'ancêtres) ou manquements aux règles rituelles (diète, relations sexuelles) sont directement identifiés comme causes des malheurs survenant pendant ou après la fête (Tableau 1).

\begin{tabular}{|l|l|l|l|}
\hline Genres de parole & $\begin{array}{l}\text { Énonciateurs } \\
\text { autorisés ou obligés }\end{array}$ & $\begin{array}{l}\text { Auditoire } \\
\text { (réel ou supposé) }\end{array}$ & Non-auditoire \\
\hline $\begin{array}{l}\text { plaisanteries et } \\
\text { grossièretés à } \\
\text { allusions sexuelles }\end{array}$ & $\begin{array}{l}\text { uniquement les } \\
\text { hommes }\end{array}$ & $\begin{array}{l}\text { les hommes, parfois } \\
\text { les femmes }\end{array}$ & $\begin{array}{l}\text { les enfants (moins } \\
\text { de 15 ans), souvent } \\
\text { les femmes }\end{array}$ \\
\hline paroles cérémonielles & initiés & les ancêtres, autres initiés & $\begin{array}{l}\text { les non-initiés, } \\
\text { femmes et enfants }\end{array}$ \\
\hline $\begin{array}{l}\text { incantations } \\
\text { murmurées }\end{array}$ & soigneurs & $\begin{array}{l}\text { divinités, esprits, } \\
\text { ancêtres }\end{array}$ & les humains \\
\hline
\end{tabular}

TABLEAU 1 - Genres de parole et types de participation supposée des sujets dans la communication.

Notons que les règles régissant la formulation et l'écoute des paroles rituelles sont plus ou moins strictes et contraignantes en fonction du type de cérémonie, du statut des partenaires ou des catégories de danses qui sont réalisées. Sans pouvoir ici présenter les différentes cérémonies dansantes, signalons néanmoins que les risques assumés sont toujours considérés comme beaucoup moins conséquents dans un bal de chontaduro que dans un rite de Yurupari ${ }^{22}$. 
Comme dans les rites funéraires, l'ensemble des sons produits lors d'un bal n'est pas uniquement destiné aux sujets qui sont rassemblés. Certains instruments sont précisément employés pour émettre des sons au-delà des rassemblements même : il s'agit des tambours manguaré et des trompes. Les manguaré sont spécialement utilisés pour émettre des messages au loin (et non pour jouer de la musique comme les trompes). Certains rythmes de tambour portent un nom et annoncent un événement précis. Par exemple, le rythme appelé kutupana annonce que les hôtes attendent leurs invités avec d'abondantes réserves de guarapo (alcool d'ananas). Quant aux trompes, les plus importantes sont évidemment celles $\mathrm{du}$ Yurupari $^{23}$ qui peuvent à la fois fournir des hauteurs de notes différentes (composer des mélodies), être entendues de loin et être déplacées durant la cérémonie ${ }^{24}$. Sans doute avec plus de rigueur que la plupart des autres instruments musicaux, ces trompes séparent ceux qui les entendent en groupes bien distincts : d'un côté, les initiés, qui peuvent les voir, les manipuler, en jouer et en connaître toute l'histoire secrète ${ }^{25}$; de l'autre, les non-initiés qui n'en ont pas le droit. En dehors des initiés qui en jouent, toutes les autres personnes qui les entendent n'ont accès qu'à un minimum d'informations : celles du moment et du lieu de mugissement des trompes (censées être accompagnées du Yurupari). Ce sont les seules informations « publiques » (au sens de « librement diffusées ») de tout le rituel. On voit bien comment le rite de Yurupari peut, au seul son de ses trompes, dissocier deux espaces : l'un « privé » ou secret et ayant le privilège de recevoir un maximum d'informations pertinentes pour les participants, l'autre « public » ou libre d'être entendu par tous, mais ne recevant qu'une information extrêmement réduite.

En dehors de ces trompes, il en existe d'autres qui n'ont rien de sacrées et peuvent être vues et touchées par n'importe qui : il s'agit des trompes luwí luwí ${ }^{26}$. Elles n'ont qu'une seule fonction : celle de se faire entendre au loin. Elles sont utilisées, soit par les hôtes pour appeler les invités humains ou non humains (ce qui, du même coup, avise également les non-invités qu'une fête est en train d'être donnée), soit par les invités pour prévenir les hôtes de leur arrivée. Ces trompes contribuent ainsi à faire savoir l'événement de la fête, mais ne fournissent (à ma connaissance) aucune autre information aux personnes non présentes.

\section{DE LA FERMETURE À L'OUVERTURE DE L'ORGANISATION SOCIALE}

On peut dire que la société indienne a atteint une nouvelle étape dans l'ouverture de son organisation sociale lorsqu'elle a modifié ses modalités propres et autonomes d'utilisation du temps et de l'espace pour les mettre en adéquation avec celles que la société dominante lui a proposé de créer pour entretenir ses rapports avec elle. En effet, des moments et des lieux ont été spécialement consacrés aux rapports des Yucuna avec l'extérieur, ce qui n'a pas manqué de 
transformer les espaces de rassemblement traditionnels en espaces publics modernes. Le véritable tournant dans la transformation de ces espaces s'est produit notamment sous l'influence d'acteurs blancs mobilisant ou faisant valoir les règles de nos institutions occidentales, principalement celles du marché, de l'Église et de l'État.

\section{Les acteurs de l'ouverture}

Sous la pression de la société dominante, les espaces à comité restreint d'autrefois ne peuvent plus se maintenir. En se transformant peu à peu, les nouveaux rassemblements libèrent la diffusion des informations, ce qui produit deux conséquences : d'une part, une perte de contrôle de la circulation des informations, d'autre part, une diminution des formes fermées de rassemblement au profit de nouvelles formes qui, elles, sont largement ouvertes.

\section{Les exploiteurs de caoutchouc ou caucheros}

Ce changement des règles du jeu a laissé des traces dans la littérature orale, notamment dans une histoire que j'ai recueillie sur l'arrivée des Blancs (Fontaine 2001, pp. 656-726). Résumons la version des événements narrée par Milciades Yucuna et recueillie en 1999. Après avoir réduit en esclavage plusieurs groupes yucuna et matapi en haut du Miriti, les premiers caucheros suscitèrent une rébellion indigène au cours de laquelle plusieurs d'entre eux moururent. Avertie par quelques rescapés, une commission répressive vint ensuite régler ses comptes avec les Indiens rebelles. Mais, au lieu de déclencher une guerre de vendetta conforme aux conflits traditionnels indigènes, la domination des Blancs par les $\operatorname{armes}^{27}$ interdit aux indigènes toute forme de violence à leur encontre ou intertribale. C'est alors que les caucheros instaurèrent le travail forcé comme nouvelle manière de résoudre les délits de meurtre ou de vol.

D'abord, ils s'adressèrent à l'un des maîtres de maloca et le nommèrent « capitaine » (espagnol vernaculaire capitán), ce qui lui fit assumer une charge de responsable et d'intermédiaire entre les groupes indigènes et leur nouvelle autorité dominante. Ensuite, ils forcèrent les Indiens à leur raconter les faits et à leur donner les raisons qui les avaient poussés à tuer leurs associés. Puis, ils obligèrent le capitaine à faire venir tous les membres des autres familles impliquées dans la rébellion. Le maître de maloca transmit ses paroles à ses frères pour qu'ils aillent les répéter aux autres. Au fur et à mesure que les familles vinrent se rassembler auprès du capitaine, les Blancs renouvelèrent leurs interrogatoires et leurs réprimandes, toujours sous la menace de leurs armes ${ }^{28}$. Ils notèrent par écrit les déclarations des indigènes et recensèrent toutes les personnes rassemblées. Ils leur firent rendre les marchandises (fusils, vêtements) volées lors de la rébellion, réclamèrent le prix de celles qui avaient été perdues ou abîmées et fixèrent une somme totale. Enfin, quand ils revinrent, ils leur donnèrent de 
nouvelles marchandises et leur déclarèrent plus tard qu'il fallait les régler en travaillant le latex...

Tels qu'ils ont été décrits par Milciades Yucuna, ces premiers rassemblements ordonnés et organisés par les Blancs changèrent radicalement les conditions traditionnelles de communication et d'échange. En effet, pour la première fois, on résolut un conflit localisé et relativement refermé sur lui-même en l'ouvrant et en le désamorçant publiquement. D'une violence clandestine difficilement justifiable d'un point de vue extérieur, on passe donc à un autre type de violence, relativement plus acceptable sur la scène publique. On instaure des conditions contextuelles nouvelles, inconnues auparavant dans l'organisation sociale indigène.

1) L'apparition du statut de capitaine assumant de nouvelles charges et responsabilités pour transmettre et diriger la communication entre les différents groupes.

2) L'utilisation de l'écrit pour rédiger des documents administratifs (déclarations indigènes, recensements). Ces documents permettent de favoriser la circulation et la conservation des informations, non seulement au sein de la commission, mais encore vis-à-vis des autres instances dominantes (en particulier l'État).

3) La mise en place d'un nouveau règlement permettant de « régler » les actes de violence par de l'endettement marchand. Les victimes tuées dans la rébellion et les marchandises volées ne sont pas réglées par de nouveaux actes de violence similaires infligés aux familles des coupables, mais par un « prix » que ces derniers peuvent (en principe) compenser par leur labeur.

Cela dit, les conditions de diffusion de l'information restent encore loin d'être idéales, en raison de l'absence d'intervention et de surveillance de représentants de l'État (en tant qu'instance de domination légitime et indépendante) et du manque de connaissance des Indiens : que ce soit en matière de comptes (pour l'évaluation de leur dette ou des prix des marchandises), d'alphabétisation (pour la lecture et la rédaction des documents administratifs) ou, encore, de compréhension et d'emploi de l'espagnol. Ces conditions changeront avec l'arrivée des corregidores (représentants administratifs de l'État) et des missionnaires.

\section{Les représentants de l'État}

Les premiers représentants de l'État constituèrent une seconde vague d'influence qui vint déterminer encore davantage les nouvelles conditions de communication et d'échange imposées aux Indiens.

Après leur avoir enseigné à collecter le lait d'hévéa, les exploiteurs de caoutchouc ne tardèrent pas à les battre et à les fouetter lorsqu'ils n'étaient pas 
satisfaits de leur travail. Au point qu'à partir de la fin des années 1920, l'État décida d'exercer une influence et un contrôle dans la région, après avoir été informé des excès de violence de la part des caucheros de Campoamor (Domínguez et Gómez 1990).

Selon Milciades Yucuna, le premier corregidor serait, à son tour, intervenu en interrogeant les Indiens sur leurs conditions de travail avec les caucheros. Il interrogea également leur patron, Oliverio Cabrera, et fit valoir son mandat du gouvernement l'autorisant à surveiller les conditions de travail. Puis il les rassembla tous ensemble pour un dernier interrogatoire public et interdit aux caucheros de battre les Indiens ou de les faire travailler sans les payer à un prix légitime pour le remboursement de leurs dettes.

L'intervention du corregidor accentua encore l'ouverture des rassemblements qui se faisaient jusque-là uniquement entre indigènes et caucheros. Entre en scène une nouvelle instance de domination qui, en dernier ressort, peut se montrer supérieure à toutes les autres : celle de l'État. Les nouvelles conditions contextuelles définissant l'espace public encore en transformation sont posées par la présence de corregidores qui, en tant que représentants de l'autorité dominante, sont chargés 1) de déclarer ses règles (interdire le travail forcé ou toute violence illégitime), 2) de surveiller leur application et 3) de transmettre les informations ayant rapport au respect de ces règles. Par la suite, tous les cahiers de compte seront « ouverts" ou « rendus publics» dans la mesure où ils devront être régulièrement contrôlés par les corregidores ${ }^{29}$.

\section{Les missionnaires}

Une troisième vague d'influence extérieure s'impose à la fin de la guerre colombo-péruvienne, lorsque les missionnaires catholiques s'installent à La Pedrera en créant, en 1934, un orphelinat pour accueillir les enfants des familles décimées par le conflit et les épidémies (Van der Hammen 1992, pp. 41-43). Rapidement, l'installation devient un internat dans lequel les Indiens du Miriti se voient contraints de laisser leurs enfants (de six à douze ans) environ dix mois de l'année. En 1949, la mission catholique fait construire un nouvel internat au Miriti, au niveau des rapides de Jariyé, sur l'ancien lieu de déchargement d'Oliverio Cabrera (el depósito). Les missionnaires enseignent aux Indiens non seulement à lire et à écrire, mais surtout à compter pour défendre au mieux leurs intérêts dans les échanges. Ils contribuent aussi à faire passer dans les mœurs l'usage de la monnaie dans la région. Très tôt, ils apprennent aux Indiens à se servir d'unités de compte en commençant par utiliser des jetons et apportent une source de revenus permanents qui initie la monétarisation de l'économie locale. Mais, dans bien des cas, l'autorité des missionnaires est jugée inacceptable. En effet, l'éducation missionnaire gêne ou remet en cause l'ensemble de la culture indigène en la dévalorisant, en la ridiculisant ou, encore, en détournant les enfants de l'enseignement traditionnel ${ }^{30}$. L'usage d'une langue indigène est 
interdit à l'internat et sévèrement réprimé. De plus, les missionnaires tentent d'éradiquer les cérémonies dansantes (le rite de Yurupari est diabolisé).

Ainsi, l'action des missionnaires tend doublement à transformer la société indigène, d'une part, en lui donnant davantage de moyens pour son ouverture, d'autre part, en sapant ceux qui, au contraire, contribuaient à sa fermeture. D'un côté, les missionnaires offrent progressivement aux Indiens certains moyens nécessaires à leur entrée en tant qu'acteurs à part entière dans la société blanche, ce qui leur permettra ensuite d'acquérir et de revendiquer des droits équivalents à tous les autres citoyens colombiens et ainsi d'intervenir peu à peu sur la scène publique. D'un autre coté, ils condamnent et interdisent la plupart des pratiques et rites indigènes méconnus ou non reconnus officiellement, c'est-à-dire les rassemblements privés, les formes de communication secrètes et l'usage de langues inconnues des Blancs qui empêchent la société dominante d'exercer son pouvoir de surveillance ou d'accès à l'information. Nous verrons que ces pratiques n'acquerront progressivement une certaine légitimité que lorsque les anthropologues auront permis de mieux les faire connaître et reconnaître publiquement.

\section{Les nouveaux espaces de rassemblement}

Les premiers lieux de rassemblement instaurés par les Blancs furent d'emblée des lieux d'un genre nouveau. Ainsi Campoamor devint le plus grand campement d'exploitation de caoutchouc de toute la région en allant jusqu'à réunir un millier d'indigènes provenant de groupes ethniques très divers et jamais rassemblés auparavant. Un tel mélange pluriethnique altéra définitivement l'ensemble des relations intertribales et de parenté, en raison des nouvelles formes de résidence et d'alliance suscitées par les patrons blancs (Van der Hammen 1992, p. 35). Certaines informations " publiques » auraient d'ailleurs été véhiculées à partir de ce lieu, à commencer par la réputation de « meurtriers » qu'avaient les Péruviens de la Casa Arana, une réputation fortement entretenue par Cabrera et son personnel pour que les populations indigènes viennent se réfugier auprès d'eux (ibid., p. 34).

À partir de la guerre colombo-péruvienne (1930-1933), d'autres espaces prirent définitivement le relais de Campoamor en tant que lieux de concentration des populations et de rapports privilégiés avec la société blanche. Tout d'abord Araracuara, où la plupart des collecteurs de Campoamor furent envoyés pour construire une piste d'atterrissage nécessaire aux actions militaires ${ }^{31}$.

À la fin de la guerre, le village frontalier de La Pedrera commença à prendre forme, non uniquement à proximité de la base militaire ${ }^{32}$, mais également autour de l'internat et du corregimiento nouvellement créés. De là, les missionnaires cherchèrent à entretenir des relations de plus en plus régulières avec les Indiens. Ils les attiraient en créant de petits magasins (tiendas) qui concurrençaient fortement les prix des marchandises offertes par les caucheros aux collecteurs. De 
plus, ils les convertissaient au catholicisme en organisant publiquement des messes et des offices. La Pedrera ne connaîtra son véritable essor démographique qu'à partir du milieu des années 1970, car, en raison de l'interdiction de l'exploitation du caoutchouc et des fourrures par INDERENA ${ }^{33}$ en 1974, l'économie de la région se détourne alors de l'extraction forestière et se recentre sur la pêche commerciale. À La Pedrera, le développement sera également favorisé par la création d'une piste d'atterrissage en 1975 (Rodríguez Fernández 1991). Beaucoup d'indigènes vont dès lors se rapprocher de cette zone urbaine afin de trouver du travail et d'avoir accès à des marchandises devenues pour eux indispensables. À mesure que ces derniers viennent y travailler, plusieurs anciens caucheros et commerçants cessent leurs déplacements itinérants sur les fleuves et ouvrent leurs premiers magasins et chambres froides dans le village.

Depuis cette époque, le village apparaît comme un lieu typiquement occidental dans lequel les Indiens se retrouvent dans des conditions radicalement différentes de celles qui sont définies traditionnellement et qui caractérisent les espaces publics modernes. Toutes les rues, toutes les places ont pour particularité de laisser leur accès ouvert à tout le monde ${ }^{34}$. Chacun peut même y observer, dire $^{35}$ ou faire des choses interdites, voire impensables, dans les situations indigènes traditionnelles. Une multitude d'objets, d'actes et de formes d'expressions de toutes sortes (marchandises, musiques, spectacles, sons de cloches, hauts parleurs, etc.) s'y donnent d'ailleurs à voir et à entendre en permanence ou cherchent à attirer au mieux l'attention des passants. En outre, différents services publics, pris en charge par l'État, sont censés y être mis gratuitement à la disposition de tous, en principe pour protéger chacun de toute violence illégitime (grâce à la police ou à l'armée) ou pour lui apporter certaines ressources considérées comme essentielles pour son niveau de vie : des soins médicaux (grâce à un centre de santé au début des années 1970) et une éducation (grâce à une école laïque au début des années 1990).

\section{LES ASSEMBLÉES COMME MOTEURS DE TRANSFORMATION D'UNE SOCIÉTÉ OUVERTE}

Une fois ouverte aux premiers représentants de l'économie de marché, de l'Église et de l'État, la société yucuna ne semble plus pouvoir se refermer ${ }^{36}$. De là une réorganisation radicale, non plus autocentrée, mais exocentrée, ce qui revient à dire que la société ne se tourne plus essentiellement sur elle-même, mais vers l'ensemble du monde englobant qui la domine. Dans ce processus d'ouverture vers l'extérieur, les nouveaux espaces publics se sont révélés être de puissants moteurs de transformation de la société, les groupes indiens étant de plus en plus habitués à faire appel aux intervenants extérieurs et à les laisser constater leurs modalités de fonctionnement, pour mieux bénéficier des moyens technologiques et des avantages sociaux promis par la société moderne. 
En tant que situations très représentatives de ce qu'il faut entendre de nos jours par « espace public » chez les Yucuna, les assemblées présentent un grand nombre de caractéristiques similaires ou comparables aux espaces publics de la société contemporaine ; elles sont aujourd'hui couramment intégrées au sein de l'organisation sociale indigène et forment des lieux de communication et d'échange très dynamiques.

Au tout début des années 1970, les Yucuna et les autres groupes exogamiques voisins (Matapi, Tanimuca, Letuama) ne faisaient pas encore de réunions pour discuter de leurs problèmes vis-à-vis des Blancs. Ils n'en parlaient que dans des situations traditionnelles et, surtout, à l'occasion des cérémonies dansantes (Pierre-Yves Jacopin, communication personnelle). Les assemblées indigènes semblent avoir été adoptées un peu plus tard, vers le milieu des années 1970 - précisément au sein de la maloca -, à l'initiative de quelques anthropologues qui avaient décidé de s'engager ou de s'impliquer dans la cause indigène. En effet, devant la misère et le désastre ethnocidaire des populations indigènes provoqués par l'exploitation des caucheros et l'influence des missions (Jacopin 1971), beaucoup d'anthropologues (Von Hildebrand et Elizabeth Reichel, entre autres) décident au début des années 1970 de réaliser une " anthropologie d'urgence » avant que ces cultures ne disparaissent complètement. Rapidement, ceux-ci se sont présentés comme d'ardents défenseurs des indigènes, en se montrant hostiles à l'égard des caucheros, des commerçants et des missions, jugés responsables de l'ethnocide. Cela les a conduits à partager leurs idées avec celles des Indiens, en leur proposant de considérer d'un point de vue sociologique et politique leurs rapports avec les Blancs.

Ainsi, les anthropologues ont largement participé à la réorganisation des communautés indigènes autour de nouveaux types d'espaces sociaux : les réunions communautaires et les congrès intercommunautaires. Ces rencontres étaient entièrement nouvelles pour les indigènes du Miriti-Parana et du Bas Caqueta, puisqu'ils ne s'étaient encore jamais rassemblés spécialement pour discuter de leurs problèmes communs, en vue de les résoudre pacifiquement selon les modalités offertes par la société dominante.

Je n'exposerai pas davantage l'émergence des activités véritablement socio-politiques, car cela demanderait une étude spécifique qui n'est pas notre propos dans cet article ${ }^{37}$. Je me limiterai à analyser ce qui caractérise les assemblées en tant qu'espace public. Pour cela, il convient de dégager les conditions contextuelles récurrentes qui déterminent leurs situations interlocutives. Je présenterai ces conditions à partir des réunions et congrès auxquels j'ai assisté à de multiples reprises ${ }^{38}$.

Identifions les règles de ces assemblées, à commencer par les obligations et les interdits susceptibles d'entraîner des sanctions (de toutes sortes) en cas de transgression (unique ou répétée). Les réunions communautaires et les congrès intercommunautaires fonctionnent selon des règles que l'on 
retrouve à des degrés d'observance divers ${ }^{39}$ dans la plupart des assemblées actuelles, à savoir :

1) L'obligation d'annonce. Toute assemblée doit être déclarée à ses participants potentiels.

2) L'obligation de présence. Les participants ont l'obligation d'être présents du début à la fin : soit tous les membres de la communauté pour une réunion, soit tous les délégués de chaque communauté pour un congrès. Toute absence, retard ou départ anticipé, non justifié, peut être sanctionné.

3) L'obligation de direction de l'assemblée. Toute assemblée doit avoir un ou plusieurs responsables chargés d'organiser l'assemblée et de se répartir les différentes tâches nécessaires aux activités prévues.

4) L'obligation de finalité de l'information. Aucune assemblée ne peut avoir lieu à vide, ou sans objet. Toute assemblée nécessite que les échanges de parole et les décisions soient en rapport avec des objectifs donnés. Il revient donc obligatoirement aux responsables d'annoncer l'ordre du jour et de proposer des points faisant l'objet d'une discussion collective.

5) L'obligation pour tous de comprendre. Chacun doit écouter et suivre, autant qu'il le peut, ce qui se dit. Il doit poser des questions ou demander une traduction dans sa langue s'il n'entend pas ou ne comprend pas les informations formulées en public. Une fois les décisions et actes d'une assemblée pris, toutes les personnes présentes sont censées les avoir entendus et compris.

6) L'interdiction de perturber l'organisation de l'assemblée et son déroulement, que ce soit par des gestes, du bruit ou des paroles inopportunes.

Si ces différentes règles ne sont pas absolument nécessaires pour identifier un espace public, on remarque néanmoins qu'elles lui sont souvent associées, ou qu'elles impliquent d'autres règles qui, elles, sont nécessaires. Remarquons que la règle 5 (l'obligation pour tous de comprendre) implique la permission ${ }^{40}$ d'écouter et d'observer, une règle que j'avais déjà repérée dans d'autres espaces publics, notamment ceux du village de La Pedrera. Mais ici, toutes ces règles qui sont strictes, obligations et interdictions (susceptibles d'être sanctionnées) ont pour finalité de forcer un maximum de gens à coordonner et à transmettre efficacement leurs discours en fonction d'objectifs prédéfinis. De par la rigidité de ces règles, toute parole énoncée acquiert un pouvoir et une portée qu'elle n'a pas dans d'autres contextes. Constituer un « public » n'est plus une liberté ou un droit ${ }^{41}$, mais une contrainte. D'où la mauvaise volonté et la lassitude de beaucoup de Yucuna se plaignant de devoir aller à ces réunions.

D'autres règles plus souples, des permissions cette fois ${ }^{42}$, forment aussi des conditions caractéristiques des espaces publics. L'une est interne à l'assemblée :

7) Le droit à la parole de chacun des participants devant l'ensemble des personnes rassemblées. En principe, tous les participants ont la permission de prendre la parole devant tout le monde, mais chacun doit attendre qu'elle lui 
soit donnée au moment opportun par l'un de ses modérateurs (capitaine ou secrétaire), en fonction de l'ordre du jour et de l'organisation des communications en assemblée.

Deux autres règles concernent les communications externes à l'assemblée :

8) Le droit de diffuser les informations communiquées à l'assemblée hors de celle-ci.

9) Le droit d'expression et de critique à propos des actes de l'assemblée.

Par ces deux dernières règles, la communication orale, d'abord restreinte à l'espace occupé par les membres de l'assemblée, est autorisée à s'étendre hors de celle-ci, entraînant d'autres paroles à son propos. Ainsi les informations communiquées peuvent susciter des réactions diverses (débats, contestations, protestations) et alimenter de nouvelles assemblées.

Quoique banales dans nos sociétés modernes, ces neuf règles communes aux communications en assemblée n'en sont pas moins exceptionnelles chez les Yucuna. Comparons-les, une à une, à leurs quatre types de situation interlocutive traditionnelle (Fontaine 2001, 2002) : la vie domestique, les visites, les mingas (travaux coopératifs) et les cérémonies.

Dans le tableau ci-dessous (Tableau 2), on remarque que les seules règles communes aux assemblées et aux situations traditionnelles sont les obligations d'annonce et de présence, toujours en vigueur dans les travaux collectifs et les cérémonies. Dans la vie domestique et les visites, aucune annonce, aucune présence n'est obligatoire pour les participants potentiels. Dans les quatre types de contexte traditionnel, les informations communiquées ne sont pas obligatoirement dirigées par un ou plusieurs responsables. En outre, elles ne sont généralement pas orientées vers un but. Même si certaines peuvent l'être, elles ne le sont qu'à certains moments pour certaines personnes et ne concernent pas l'ensemble des individus présents. Toutes les personnes regroupées dans ces situations traditionnelles ne sont pas obligées en permanence de comprendre tout ce qui se dit, d'ailleurs beaucoup d'entre elles n'y sont même pas autorisées (les femmes et les non-initiés ne pouvant écouter les secrets relatifs au chamanisme ou au Yurupari). Les personnes réunies en situations traditionnelles ne s'interdisent pas de parler ou de s'activer en même temps que les autres. D'où les brouhahas et les interruptions d'activité ${ }^{43}$, très fréquents dans ces contextes.

En ce qui concerne les droits propres aux espaces publics, on constate que ceux-ci ne sont en aucun cas valables d'une manière générale dans les types de contexte traditionnels. Au niveau interne, s'il peut arriver que des personnes parlent à l'attention de toutes les autres réunies, cela ne peut jamais se prolonger en dehors de moments précis, le plus souvent réservés à quelques privilégiés dans certaines situations ritualisées (récit d'un narrateur lors d'une veillée, diagnostic d'un soigneur sur une maladie, etc.). 


\begin{tabular}{|l|l|c|c|c|c|c|}
\hline & RèGLes & DoMESTIQUE & VISITE & MINGA & CÉRÉMONIE & ASSEMBLÉE \\
\hline 1 & obligation d'annonce & non & non & oui & oui & oui \\
\hline 2 & obligation de présence & non & non & oui & oui & oui \\
\hline 3 & $\begin{array}{l}\text { obligation de direction de } \\
\text { l'assemblée }\end{array}$ & non & non & non & non & oui \\
\hline 4 & $\begin{array}{l}\text { obligation de finalité de } \\
\text { l'information }\end{array}$ & non & non & non & oui \\
\hline 5 a & $\begin{array}{l}\text { obligation pour tous de } \\
\text { comprendre }\end{array}$ & non & non & non & non & oui \\
\hline $5 b$ & droit pour tous de comprendre & non & non & non & non & oui \\
\hline 6 & interdiction de perturbation & non & non & non & non & oui \\
\hline 7 & droit de parole devant l'assemblée & non & non & non & non & oui \\
\hline 8 & droit de diffusion extérieure & non & non & non & non & oui \\
\hline 9 & $\begin{array}{l}\text { droit d'expression et de critique à } \\
\text { propos de l'assemblée }\end{array}$ & non & non & non & non & oui \\
\hline
\end{tabular}

TABLEAU 2 - Règles appliquées selon les contextes.

$\mathrm{Au}$ niveau externe, s'il est vrai que des catégories d'informations sur les actes et propos des personnes regroupées au sein d'une maisonnée peuvent être diffusées à d'autres, ce n'est généralement pas à n'importe qui et cela ne concerne pas les domaines ritualisés, ni ceux soumis aux obligations et interdits rituels. En effet, les informations relatives à ces domaines étant les plus susceptibles d'être récupérées pour donner lieu à des actions et paroles malveillantes (ragots, malédictions), elles se doivent d'être tenues secrètes.

Sur le chemin de la liberté de circulation de l'information que l'on peut idéalement concevoir au sein d'un espace public, les assemblées offrent donc des conditions plus avantageuses que celles des contextes traditionnels, mais il convient de signaler plusieurs entraves importantes. Elles grèvent en priorité les droits des individus non autorisés à participer aux assemblées, dès lors qu'ils ne sont pas affiliés aux communautés concernées et qu'ils n'en ont pas eu l'autorisation officielle. Contrairement à d'autres lieux comme ceux des espaces publics de village, l'accès n'y est donc pas ouvert à tous. En conséquence, le droit de parole n'y est pas garanti non plus. Mais il est vrai que tout étranger peut, du moins, en faire la demande, ce qui est le plus souvent accepté, à moins que sa requête ne soit rejetée après délibération en assemblée ${ }^{44}$.

Enfin, il est important de signaler que l'espace public d'une assemblée ne saurait être un lieu propice à la circulation de n'importe quelle information. Certaines connaissances confidentielles ou secrètes ne peuvent, à aucun moment, y être exposées. Ce qui pose des problèmes récurrents aux tenants de la tradition, dès lors qu'on les oblige à expliquer leurs actes et leurs conceptions, alors même qu'ils se doivent de garder des secrets réservés au chamanisme, aux rites et à l'initiation ${ }^{45}$. 


\section{CONCLUSION}

Dans ce texte, nous avons d'abord examiné l'organisation sociale traditionnelle des Yucuna pour évaluer dans quelle mesure les situations de rassemblement (ralliements de guerre et cérémonies) peuvent être comparées aux espaces publics modernes. Leur analyse a montré qu'elles partagent certains points communs, mais qu'elles s'en distinguent néanmoins, car les individus se voient privés de leur liberté dans le cadre de la situation de communication, non seulement pour y accéder, mais encore pour y émettre ou recevoir des informations. De par ces situations, soit on a le privilège de participer à la communication, soit on en est exclu ou, encore, on y est uniquement autorisé à ne recevoir qu'une information réduite au maximum (le moment de la réalisation d'un rituel).

Avec l'arrivée des caucheros, des corregidores et des missionnaires, les vieux modèles de rassemblement sont dévalorisés, interdits ou forcés de disparaître, au profit de nouvelles situations interlocutives. Les acteurs blancs établissent de nouveaux espaces d'activités et de rencontres (campements, corregimientos, internats, villages) dans lesquels ils peuvent faire appliquer d'autres règles et d'autres moyens (écrire, calculer) que ceux des Yucuna dans leurs espaces traditionnels. Á la fois sous leur domination et sous leur protection, chacun accède à davantage de droits pour observer, s'exprimer, écouter et agir en toute sécurité et avec de nouvelles possibilités (pour s'instruire ou se soigner).

L'intrusion des anthropologues dans ce champ a encore accéléré la métamorphose de la société yucuna. Toutefois ces derniers n'attaquent pas directement l'organisation sociale interne, en condamnant par exemple les rassemblements traditionnels où se jouent des formes exacerbées de violences ou d'inégalités (par exemple entre hommes et femmes). Ils visent plutôt les relations externes de la société yucuna en cherchant à accroître son ouverture vis-à-vis de la société englobante afin de mieux légitimer les droits des indigènes à défendre leurs intérêts sociaux et leurs spécificités culturelles face aux institutions et aux interventions extérieures qui abusent de leur autorité et de leur influence. La seule manière pour les anthropologues de renforcer l'ouverture de la société indigène est d'implanter de nouveaux modèles de situations interlocutives ${ }^{46}$ telles que les assemblées qui offrent beaucoup plus d'avantages en matière de communication que toutes les autres formes d'espace public localement connues jusqu'alors. Cette fois, chacun a non seulement le droit de s'exprimer et d'observer, mais il peut aussi bénéficier du privilège d'être écouté et compris par un grand nombre de personnes, ce qui donne évidemment beaucoup plus d'efficacité et de portée à sa parole.

La société yucuna s'est donc certainement transformée, mais elle demeure « traditionnelle » tout en devenant toujours plus « moderne ». D'un côté, elle n'a jamais abandonné la reproduction d'une partie essentielle de son savoir par le 
secret (incantations, initiations par le rite du Yurupari), et c'est cela qui permet encore de légitimer ses inégalités sociales internes fondées sur des différences de connaissance et de statut. De l'autre, elle a appris qu'en devenant plus ouverte et accessible, en aménageant certains espaces publics, elle pouvait plus facilement se légitimer elle-même et réduire ses inégalités vis-à-vis des couches favorisées de la société industrielle. Deux stratégies communicatives, au lieu d'une, s'offrent à chaque Yucuna dorénavant. *

* Manuscrit reçu en janvier 2012, accepté pour publication en octobre 2012.

\section{NOTES}

L'origine de cette recherche sur les espaces publics chez les Yucuna provient d'une opération de l'équipe « Anthropologie de la Parole » du LACITO (UMR 7107), intitulée « Interlocution et espaces publics » et dirigée par Bertrand Masquelier. Plusieurs réunions furent consacrées à la présentation et à la discussion de cet article, donc je tiens à remercier les membres de cette équipe pour leurs remarques. J'en profite également pour remercier Pierre-Yves Jacopin (qui a également travaillé chez les Yucuna) qui m'a fait bon nombre de suggestions pour corriger et développer cet article.

1. Il en va de même pour beaucoup d'autres concepts empruntés à nos sociétés occidentales et, même, à nos perspectives scientifiques modernes : par exemple, sur le concept de « monnaie » et sur son existence chez les Yucuna, voir Fontaine (2002, 2008a).

2. Il est vrai qu'on aurait pu choisir de traiter les premiers contacts avec les agents extérieurs (esclaves, métis, caboclo et Blancs) ayant été identifiés par les historiens depuis le $\mathrm{XVII}^{\mathrm{e}}$ siècle (Pineda Camacho 1985 ; Llanos Vargas et Pineda Camacho 1982). Mais, en dehors du fait que les sources d'informations sont insuffisantes, ces contacts ne m'ont pas paru suffisamment intéressants pour parler d'un véritable tournant dans l'évolution des espaces publics, notamment en raison de la prépondérance des conflits intertribaux (peu propices à la diffusion des idées et des débats) qui prévalaient avant l'arrivée des premiers exploiteurs de caoutchouc.

3. On peut s'interroger sur la différence entre la parole dite privée et celle dite secrète. Je propose de définir la " parole privée » comme une parole soumise - en raison de son cadre d'énonciation - à un interdit, plus ou moins implicite, de divulguer ses informations vers d'autres personnes que les auditeurs directs. Un « espace privé » est précisément un type de cadre d'énonciation dans lequel il est conventionnellement admis que toutes les paroles qui y sont prononcées sont " privées », c'est-à-dire confidentielles par défaut à l'intérieur du groupe (contrairement à un espace public où toute les paroles y sont publiques, donc peuvent être divulguées par défaut au-delà du groupe), sauf en cas d'exception déclarée et admise communément par les interlocuteurs. Une parole secrète, quant à elle, est soumise à un même interdit de divulgation (au-delà d'un certain groupe clairement identifié), mais cet interdit n'est pas déterminé par son cadre d'énonciation. C'est pourquoi, toute parole secrète doit à un moment donné être accompagnée d'une interdiction explicite de la transmettre, du moins à certaines catégories de personnes.

4. L'espace social et symbolique de la maloca yucuna a déjà été amplement étudié (Jacopin 1972, pp. 117-130 ; Van der Hammen 1992, pp. 138-147). Je ne peux malheureusement pas y revenir dans cet article, sans m'écarter démesurément de la notion d'«espace public » selon Habermas. Notons cependant que Arhem (2001) signale, chez les Macuna (voisins des Yucuna), une division entre un « espace privé » et un « espace public », qui correspond à ce que Jacopin (ibid., p. 120) appelait une division entre un « espace familial » et un « espace étranger». Pour ma part, je considère que les termes de Jacopin sont plus appropriés, car les notions de public et de privé sont, en l'absence de définition précise, beaucoup trop connotées par nos institutions politiques occidentales. Elles peuvent laisser supposer une organisation liée à l'État et prêter à confusion. 
5. Certaines paroles peuvent très bien être adressées simultanément à de nombreuses personnes présentes, notamment lorsque le maître de maloca donne des directives. Et beaucoup de paroles, notamment rituelles (mythes, discours cérémoniels et diagnostics chamaniques), peuvent être entendues par bon nombre de personnes rassemblées, comme j'ai pu l'observer sur le terrain.

6. Cette notion de « société du secret » ne doit pas laisser entendre que toute parole est secrète dans une telle société (toute parole n'est pas non plus publique dans une "société de la publicité »), mais qu'une bonne partie des paroles sur lesquelles s'appuie l'autorité est secrète (savoir initiatique et chamanique), contrairement à la parole des hommes politiques dans nos sociétés, qui doit être rendue publique.

7. À noter que les Yucuna n'ont pas dans leur langue de terme pour dire « rassemblement » ou « réunion ». Ils disent simplement «nous allons nous regrouper » (wawakájiko). De nos jours, pour dire « congrès » ou « réunion », ils empruntent les termes espagnols (congreso, reunión).

8. Je mentionne plusieurs mythes et récits pour apporter des précisions supplémentaires à propos de ce que pouvaient constituer de tels rassemblements, étant donné qu'il n'existe, à ma connaissance, aucune autre source d'informations précises. Certes, les faits décrits dans les mythes ne peuvent pas être pris comme des faits ayant véritablement existé, car la parole mythique est toujours a-historique (Jacopin 1993-1994, p. 149). Mais il n'en reste pas moins que les thèmes et les motifs de cette parole mythique s'inspirent d'une certaine réalité de la vie sociale, c'est même cela qui la rendit, ou la rend encore, pertinente pour ses auditeurs.

9. Il s'agit d'un corpus d'une centaine de textes composés de récits, conversations et paroles cérémonielles. Toutes les informations et citations que je donne sont tirées de ce corpus.

10. Généralement, les Yucuna n'organisent pas de longs déplacements de nuit car ils sont considérés comme dangereux. Même dans les cas de poursuite en forêt, on attend généralement les premières lueurs du jour, sauf si les individus à poursuivre viennent de s'échapper et sont donc encore très proches.

11. Cette divination est présentée dans différents récits, comme le mythe de Kanumá contre les jaguars, l'histoire de Ka'marí (Ancêtre éponyme des Matapi) ou celle de Pichapatá (célèbre guerrier yucuna). Au moyen de certaines incantations, elle consiste à prévoir l'échec ou la réussite d'une bataille et le nombre de morts parmi les différentes tribus en conflit. En fonction des prévisions réalisées, un chef de guerre peut choisir la meilleure stratégie.

12. Rappelons brièvement que le Yurupari est un rituel d'initiation durant lequel les hommes jouent des flûtes et des trompes (habituellement cachées dans une rivière), sans autoriser les femmes à les voir ou à les toucher, ni même à marcher sur le sol qu'ils foulent de leurs pieds. Selon les Indiens, les femmes pourraient mourir de leurs menstruations à la simple vue de ces trompes. Les hommes doivent d'ailleurs se soumettre à d'importantes restrictions alimentaires durant de longues périodes, avant, pendant et après le rituel pour « voir Yurupari » dans les conditions prescrites par les chamanes et, ainsi, passer le cap de l'initiation cérémonielle qui leur permet de «mourir en tant qu'enfant et de revivre en adulte». Ce rituel a été décrit et analysé par divers auteurs, notamment par Mich (1994) chez les Yucuna et par Hugh-Jones (1979) chez les Barasana (de famille linguistique tucano).

13. L'histoire de Ka'marí a été traduite dans des versions relativement longues à deux reprises (Herrera Angel 1976 ; Matapi et Matapi 1997). Les précisions apportées ici proviennent d'une version que j'ai enregistrée et transcrite avec Mario Matapi.

14. Ce mythe viendrait des Letuama.

15. Кити (yucuna). Manguaré, maguaré (espagnol vernaculaire). Il s'agit d'une paire de tambours monoxyles utilisés pour la communication (Karadimas 1997). Ces tambours font environ deux mètres de long et un mètre de diamètre. On les frappe sur leur longueur avec deux massues à balle de latex.

16. Chez les Boras et Mirañas, les manguaré sont de véritables moyens de communication en raison des tons propres à leur langue qu'ils savent reproduire ou imiter au son des notes du tambour. Ce savoir musical est réutilisé pour noter les tons dans l'écriture introduite par les linguistes (Instituto Lingüístico de Verano 1989). Chez les Yucuna, chaque voyelle est prononcée sur une certaine note, aussi bien dans 
le langage courant que dans les chants, si bien que l'imitation de la langue avec un tambour monoxyle est aussi possible. Mais aucune étude précise n'a encore été faite à ce propos. Toujours est-il que plusieurs informateurs ont prétendu comprendre beaucoup de choses au seul son des manguaré, puisque la plupart des rythmes caractéristiques sont associés à des événements-types.

17. Le principe de coopération de Grice suppose que, dans tout échange conversationnel entre un locuteur et un interlocuteur, il y a un minimum d'effort coopératif entre eux, ce qui oblige le premier à respecter certaines règles conversationnelles (appelées maximes de quantité, de qualité, de relation et de manière). En supposant cela, l'interlocuteur peut déduire d'autres informations que celles qui lui ont été dites et orienter ses interprétations.

18. Sperber et Wilson considèrent que c'est uniquement le principe de pertinence (id est la maxime de relation de Grice) qui est supposé dans tout échange conversationnel et que ce seul principe permet à l'interlocuteur de réaliser un grand nombre d'inférences à partir des énoncés et des autres informations déjà connues.

19. Ces paroles sont apprises et répétées secrètement en forêt.

20. Malgré mes tentatives pour les enregistrer et les filmer in situ, il m'a été impossible de parvenir à les transcrire, même avec des informateurs expérimentés. Mais il est vrai que la situation ne s'y prêtait guère. Les locuteurs acceptent déjà difficilement la présence d'un micro ou d'une caméra, il est donc difficile de leur imposer de meilleures conditions d'enregistrement. Seuls des enregistrements effectués en particulier avec certains initiés (hors situation) ou dans des rituels plus restreints (situation de visite) se sont avérés exploitables pour la transcription. Certains ont été présentés dans ma thèse (Fontaine 2001), d'autres sont encore en cours d'étude.

21. On retrouve l'idée des incantations murmurées dans d'autres groupes de la région. Pour une étude chez les Barasana, voir Buchillet (1992).

22. Pour une description du rite de Yurupari, voir Mich (1994). Par ailleurs, j'ai décrit une version mineure de ce rite et un bal de chontaduro (Fontaine 2001, pp. 266 et 529-537).

23. Je n'ai pas été autorisé à les observer en détail. Je n'ai pu les « voir » qu'une fois de loin et dans l'obscurité de la nuit. En revanche, je les ai bien entendues comme n'importe quel non-initié. Pour une description de ces trompes chez les Barasana, voir Hugh-Jones (1979).

24. Les non-initiés restant à l'intérieur de la maloca peuvent ainsi entendre les sons des trompes s'intensifier peu à peu au fur et à mesure que les initiés se rapprochent, puis tourner autour d'eux à l'extérieur de la maloca.

25. Une part fondamentale de cette histoire est racontée dans le dernier épisode du mythe d'origine (le mythe des Karipú Lakena), dont j'ai pu transcrire et traduire deux versions auprès de deux narrateurs, Milciades Yucuna et Mario Matapi. Mais la suite n'a pu m'être révélée ; elle n'est racontée qu'aux initiés durant le rituel même.

26. Faites de balsa, elles ont une forme cylindrique et mesurent généralement entre 50 et $70 \mathrm{~cm}$ de long et de 10 à $15 \mathrm{~cm}$ de diamètre.

27. Rappelons que le gouvernement intervint une première fois dans la région en 1911, en envoyant depuis Barranquilla à La Pedrera une troupe armée dirigée par le général Gamboa, afin de freiner les plans d'expansion de la Casa Arana (célèbre compagnie péruvienne d'exploitation du latex) et de soutenir les caucheros colombiens, dont Oliverio Cabrera. Ceux-ci défendirent leurs intérêts en prêtant main forte à l'armée. À la fin du conflit, Cabrera reçut un grand nombre de fusils, et plusieurs hommes restés sur place se mirent à travailler pour lui.

28. Selon le narrateur, les Blancs menacèrent, à plusieurs reprises, les Indiens de les massacrer tous s'ils recommençaient à tuer leurs semblables.

29. Notons, cependant, qu'ils ne remettent pas en cause l'exploitation par endettement, ni même les fustigations ou les représailles des caucheros. Au contraire, ils se chargent de légitimer et de faire appliquer de telles sanctions lorsque les collecteurs n'apportent pas à leur patron la contrepartie en latex de leur dette.

30. Dans une histoire recueillie par les linguistes du Summer Institute of Linguistics, Bonifacio Matapi rapporte les paroles de son oncle, lorsque celui-ci n'avait pas voulu le laisser partir à l'internat, 
précisément pour qu'il puisse continuer à apprendre le savoir traditionnel avec lui (Matapi et Matapi 1984).

31. Un tel rapprochement entre collecteurs et militaires colombiens entraîna la diffusion d'épidémies de grippe et rougeole qui firent périr un grand nombre d'indigènes.

32. Rappelons que le village de La Pedrera fut créé en 1911 à l'occasion du premier conflit colombo-péruvien. Ce lieu n'était auparavant qu'un poste de douane.

33. INDERENA : Instituto Nacional de los Recursos Naturales y del Ambiente (Institut national des ressources naturelles et de l'environnement).

34. À La Pedrera, tous ces espaces de passage (à usage de tous et du domaine public) partagent la plupart des caractéristiques des places et des autres lieux publics d'Amérique latine, tels qu'ils ont été décrits et étudiés par Low (2000). De tels lieux constituent des espaces publics au sens d'Habermas dans la mesure où ils ont la particularité d'être des lieux de propagation d'une multitude d'informations. C'est la raison pour laquelle bon nombre d'indigènes vont très régulièrement au village, même s'ils n'ont rien de spécial à y faire. Mais ce n'est pas parce que des individus se placent dans de tels lieux, qu'ils ne peuvent pas y converser confidentiellement. Tout lieu, même s'il est utilisé spécifiquement à certaines fins, peut aussi être utilisé à d'autres... Il en est aussi ainsi pour l'espace de la maloca qui peut être tantôt domestique, tantôt festif (Jacopin 1972, pp. 117-128).

35. Il est intéressant d'indiquer que les Yucuna associent l'origine des « ragots » (yucuna chapujreji, espagnol chisme) à l'influence des Blancs. Il est vrai que les libertés de communication offertes par les conditions interlocutives occidentales apparaissent beaucoup plus favorables à l'émission et à la diffusion des ragots. Peut-être étaient-ils bien mieux contrôlés avant les premiers contacts avec les Blancs ?

36. Popper (1979, chap. 10) a remarquablement rendu compte de cette irréversibilité des « sociétés ouvertes ").

37. Pour plus d'informations sur les évolutions socio-politiques des Yucuna depuis les années 1970, je renvoie le lecteur à ma thèse (Fontaine 2001, pp. 290-299).

38. Entre juillet 1997 et septembre 2011, sur un total de quarante-six mois de terrain, j'ai assisté à une trentaine de réunions communautaires et à deux congrès intercommunautaires.

39. Lorsqu'une règle stricte n'est plus rigide, on dit qu'elle « s'affaiblit » et plus personne n'est (ou ne se sent) obligé de sanctionner le sujet irrespectueux de la règle. Ce qui était obligatoire est alors valorisé et ce qui était interdit devient dévalorisé (Fontaine 2007).

40. Les implications entre règles font référence à la logique déontique de Von Wright décrite dans son fameux article de 1951. Pour une présentation commentée et développée, voir Kalinowski (1996).

41. On considère qu'une liberté est différente d'un droit dans la mesure où un droit est toujours garanti par une autorité supérieure chargée de sanctionner toute autre autorité interdisant et punissant ce qu'elle protège en tant que droit. Quant à la liberté, elle ne requiert aucune autorité supérieure pour être garantie, parce qu'aucune autorité ne la menace. Cela n'empêche pas de parler idéalement de « liberté », précisément quand elle est menacée.

42. Le permis (ou le droit) constitue l'une des quatre règles de la logique déontique de Von Wright (1951) avec le facultatif, l'interdit et l'obligatoire. Le permis et le facultatif sont pour moi des règles « non strictes » (Fontaine 2001, pp. 40-41) dans la mesure où elles ne sont pas susceptibles d'être trangressées et, donc, sanctionnées, qu'on les accomplisse ou non, contrairement à l'interdit et l'obligatoire.

43. Si un homme ou une femme a prévu de réaliser une certaine tâche à un moment de la journée, n'importe qui (un nouveau visiteur, un enfant venant de se blesser, etc.) peut l'obliger à retarder cette tâche. Même lorsque les soigneurs prononcent leurs incantations, ils peuvent le faire à proximité de bavardages et être fréquemment interrompus sans manifester aucune gêne.

44. Il est fréquent que les indigènes de passage dans leur famille souhaitent assister, voire parler aux réunions. Ils peuvent ou non être acceptés en fonction de divers critères tels que leur statut, leur sagesse (d'ancien), leurs antécédents (comme fauteurs de troubles) ou leurs compétences reconnues par rapport aux propos du moment.

45. Lors d'une réunion ayant eu lieu le 2 août 2006 à Camaritagua (communauté indigène juste à côté du village de La Pedrera), on avait ainsi interrogé les anciens sur les modalités traditionnelles de 
sanctionner les jeunes, pour éviter de recourir systématiquement au code correctionnel de la police de La Pedrera. Or les anciens étaient bien en peine de répondre, car ces modalités reposaient principalement sur les malédictions et le rite de Yurupari (Fontaine 2008b).

46. La place a manqué dans cet article pour traiter des autres situations interlocutives que les anthropologues expérimentent et suscitent auprès de leurs hôtes et informateurs indigènes (Masquelier et Siran 2000 ; Petrich 2000). On peut considérer que la plupart de leurs activités professionnelles (du terrain aux communications, du journal de terrain aux publications, des enregistrements audio à la vidéo, etc.) contribuent également à la transformation des espaces publics dans la mesure où elles exposent des informations qui, sans eux, demeureraient inconnues, et réorganisent toute une série de lieux sociaux afin de les faire connaître ou de les remanier ouvertement.

\section{RÉFÉRENCES CITÉES}

ArHem Kaj

2001 «From longhouse to village. Structure and change in the Colombian Amazon », in Laura M. Rival et Neil L. Whitehead (éd.), Beyond the visible and the material. The Amerindianization of society in the work of Peter Rivière, Oxford University Press, Oxford, pp. 123-157.

Austin John Langshaw

1970 Quand dire c'est faire, Le Seuil, coll. « Essais », Paris.

Buchillet Dominique

1992 "Nobody is there to hear: Desana therapeutic incantations ", in Jean E. Matteson Langdon et Gerhard Baer (éd.), Portals of power : shamanism in South America, University of Mexico Press, Albuquerque, pp. 211-230.

Domínguez Camilo Arturo et Augusto Gómez

1990 La economía extractiva en la Amazonia colombiana : 1850-1930, Tropenbos/ COA, Bogota.

FONTAINE Laurent

$2001 \quad$ Paroles d'échange et règles sociales chez les Indiens yucuna d'Amazonie colombienne, thèse de doctorat, Iheal/Paris III, Paris [http://tel.archivesouvertes.fr/docs/00/59/66/37/PDF/These, consulté le 18/07/2013].

2002 «La monnaie, une modalité d'échange parmi d'autres chez les Indiens yucuna d'Amazonie colombienne », Journal des anthropologues, 90-91, pp. 171-188.

2007 «Logiques modales et anthropologie : des règles à la parole chez les Indiens yucuna d'Amazonie colombienne », L'Homme, 184, pp. 131-154.

2008a "Les monnaies chez les Yucuna d'Amazonie colombienne : de la coca à mâcher au peso ", in Éveline Baumann, Laurent Bazin, Pépita OuldAhmed, Pascale Phelinas, Monique Selim et Richard Sobel (éd.), L'argent des anthropologues, la monnaie des économistes, L'Harmattan, Paris, pp. 135-166.

2008b «Les nouvelles interactions entre Yucuna et intervenants extérieurs (Colombie amazonienne) », Bulletin de la Société suisse des Américanistes, 70, pp. 49-58 [http://www.ssa-sag.ch/bssa/pdf/bssa70_08.pdf, consulté le $10 / 01 / 2012]$. 
2010a "Agents" ou "patients"? De l'agentivité des chamanes yucuna d'Amazonie colombienne », Ateliers du LESC, 34 [http://ateliers.revues. org/8526, consulté le 15/01/2012].

2010b « Histoire de Kanumá », Programme Archivage, LACITO/CRDO, Villejuif [http://crdo.risc.cnrs.fr/data/fontaine/YCN_KANUMA.pdf, consulté le 20/01/2012].

2011 «Les cours d'eau dans les incantations chamaniques des Indiens yucuna (Amazonie colombienne) », Journal de la Société des Américanistes, 97 (1), pp. 119-149.

Grice Herbert Paul

1979 «Logique et conversation », Communications, 30, pp. 57-70.

HABERMAS Jürgen

1978 L'espace public. Archéologie de la publicité comme dimension constitutive de la société bourgeoise, Payot, Paris.

HerRera ANGel Leonor

1975a «Kanuma: un mito de los Yukuna Matapi », Revista colombiana de antropología, 18, pp. 383-415, Bogota.

1975b "Yurupari y las mujeres », Revista colombiana de antropología, 18, pp. 417-434, Bogota.

1976 «El nacimiento de los Matapi », Revista colombiana de antropología, 20, pp. 201-280, Bogota.

HuGH-Jones Stephen

1979 The Palm and the Pleiades: initiation and cosmology in Northwest Amazonia, Cambridge University Press, Cambridge.

Instituto Lingüístico de Verano

1989 Bora, vol. 3. El manguare facilita la escritura del Bora, coll. « Literaria y Cultural », Pucallpa (Perú).

JACOPIN Pierre-Yves

1971 "Contribution au débat sur l'ethnocide des Indiens d'Amazonie », in Robert Jaulin (éd.), Le Livre Blanc de l'ethnocide en Amérique, Fayard,

1972 «Habitat et territoire yucuna », Journal de la Société des Américanistes, 61, pp. 107-139.

1981 La parole générative de la mythologie des Indiens Yukuna, thèse de doctorat, université de Neuchâtel, Neuchâtel.

1993-1994 «Dynamique de la parole sociale en Amérique latine», Bulletin de la Société suisse des Américanistes, 57-58, pp. 147-159.

KALINOWSKI Georges

1996 La logique déductive, PUF, Paris.

KARADIMAS Dimitri

1997 Le corps sauvage. Idéologie du corps et représentations de l'environnement chez les Miraña d'Amazonie colombienne, thèse de doctorat, université de Paris X, Nanterre. 
Llanos Vargas Héctor et Roberto Pineda Camacho

1982 Ethnohistoria del Gran Caqueta, Finarco/Banco de la República, Bogota.

Low Setha M.

2000 On the plaza. The politics of public space and culture, University of Texas Press, Austin.

Matapi Carlos et Bonifacio MATAPI

1984 Jupimi i’imacaño yucuna. La historia de nuestros antepasados en yucuna y español, Editorial Townsend, Lomalinda.

Matapi Carlos et Uldarico MataPI

1997 Historia de los Upichia, Tropenbos, Bogota.

MASQuelier Bertrand et Jean-Louis SiRAN (éd.)

2000 Pour une anthropologie de l'interlocution. Rhétoriques du quotidien,

L'Harmattan, coll. « Logiques sociales », Paris.

Mich Tadeusz

1994 "The Yuruparí complex of the Yucuna Indians: the Yuruparí rite», Anthropos, 89, pp. 39-49.

Petrich Perla

2000 "Diálogos entre el antropólogo y el informante », in Aurore Monod Becquelin et Philippe Erikson (éd.), Les rituels du dialogue, Société d'ethnologie, Nanterre, pp. 347-363.

Pineda Camacho Roberto

1985 Historia oral y proceso esclavista en el Caquetá, Finarco/Banco de la República, Bogota.

POPPER Karl

1979 La société ouverte et ses ennemis : l'ascendant de Platon, vol. 1, Le Seuil, Paris [1945].

Propp Vladimir

1983 Les racines historiques du conte merveilleux, Gallimard, Paris.

RodRíGUEZ FERNÁNDEZ Carlos Alberto

1991 Bagres, malleros y cuerderos en el bajo río Caquetá, Tropenbos, Bogota.

RuMSEY Alan

2009 "L'anthropologie a-t-elle besoin de sa propre pragmatique ? », in Carlo Severi et Julien Bonhomme (éd.), Paroles en acte, L’Herne, coll. « Cahiers d'anthropologie sociale » 5, Paris, pp. 43-62.

SCHACKT Jon

1994 Nacimiento Yucuna. Reconstructive ethnography in Amazonia, Université d'Oslo, Oslo.

SEVERI Carlo

2009 «La parole prêtée. Comment parlent les images », in Carlo Severi et Julien Bonhomme (éd.), Paroles en acte, L'Herne, coll. « Cahiers d'anthropologie sociale » 5, Paris, pp. 11-41. 
SPERBER Dan et Deirdre WILSON

1989 La pertinence. Communication et cognition, Éditions de Minuit, Paris.

VAN DER Hammen Maria Clara

1992 El manejo del mundo, Tropenbos, Bogota.

VON WRIGHT Georg Henrik

1951 «Deontic logic », Mind, 60, pp. 1-15. 